\title{
IMAGE PRE-PROCESSING FOR OPTIMIZING AUTOMATED PHOTOGRAMMETRY PERFORMANCES
}

\author{
G. Guidi ${ }^{*}$, S. Gonizzi, L.L. Micoli \\ Department of Mechanical Engineering, Politecnico di Milano, via la Masa 1, 20156, Italy \\ (gabriele.guidi, sara.gonizzi, laura.micoli)@polimi.it
}

Commission V, WG4

KEY WORDS: Automated Photogrammetry, SFM, Image Matching, Polarizing filter, HDR, Tone mapping, Shiny materials, Dark materials, Cultural Heritage, image-based modeling performances.

\begin{abstract}
:
The purpose of this paper is to analyze how optical pre-processing with polarizing filters and digital pre-processing with HDR imaging, may improve the automated 3D modeling pipeline based on SFM and Image Matching, with special emphasis on optically non-cooperative surfaces of shiny or dark materials. Because of the automatic detection of homologous points, the presence of highlights due to shiny materials, or nearly uniform dark patches produced by low reflectance materials, may produce erroneous matching involving wrong 3D point estimations, and consequently holes and topological errors on the mesh originated by the associated dense 3D cloud. This is due to the limited dynamic range of the 8 bit digital images that are matched each other for generating 3D data. The same 256 levels can be more usefully employed if the actual dynamic range is compressed, avoiding luminance clipping on the darker and lighter image areas. Such approach is here considered both using optical filtering and HDR processing with tone mapping, with experimental evaluation on different Cultural Heritage objects characterized by non-cooperative optical behavior. Three test images of each object have been captured from different positions, changing the shooting conditions (filter/no-filter) and the image processing (no processing/HDR processing), in order to have the same 3 camera orientations with different optical and digital pre-processing, and applying the same automated process to each photo set.
\end{abstract}

\section{BACKGROUND}

\subsection{Photogrammetry with polarizing filters}

Light is generally idealized as a sequence of photons, each one representing a short piece of electromagnetic wave. The direct sunlight has an orientation randomly varying, therefore is not polarized, but the light coming from the blue sky (i.e. reflected from the water molecules suspended in air), is characterized by a specific orientation determined by the dipole structure of water $\left(\mathrm{H}_{2} \mathrm{O}\right)$. When looking at such light from a direction at right angles with respect to the sun rays, such polarization is maximized and a polarizing filter, if rotated orthogonally to the polarizing orientation, will filter out the polarized component of skylight darkening the sky. The landscape below it and the clouds, will be less affected, giving an image with a darker and more dramatic sky, emphasizing the contrast with the clouds (Goldberg, 1992). Although such use of polarizing filters is well known from the very early stages of photography as a way for reducing the strong sky luminance or for eliminating reflections from glasses or stretches of water (Feininger, 1954), only few works have been published about the use of filtering with polarizers in photogrammetry. In aerial applications (Paine et al., 2012) have demonstrated that polarizing filters improve imaging on water and allow to penetrate haze, while in closerange photogrammetry they have been used for precise detection of shiny aluminum structures and retroreflective targets in aerospace industry (Wells et al., 2005).

No systematic analysis on SFM/Image Matching application seems to be available in the literature, neither for general purpose nor for Cultural Heritage applications.

\subsection{Photogrammetry with HDR}

The dynamic range of real-life scene, intended as the ratio between the highest and the lowest light intensity in the scene framed by a camera, can reach huge values, up to 500,000:1 (Debevec and Malik, 1997).

In general a digital camera is not able to manage such wide range of light intensities due to the limited dynamic range of its image sensing stage (the only still analog section of each digital camera) that is not able to detect currents below a minimal level indicated as the "noise floor", and saturates if the light overcome a maximum level defined by current amplifier before the A/D conversion stage (Holst, 1996; Robertson et al., 2013). In addition, the limited number of bits associated to each pixel reduces the number of levels in which each picture element is digitized, determining the quantity of details that, for a certain exposure, each camera is able to simultaneously represent in the darker and in the lighter areas of an image. Such kind of images (i.e. those usually generated by a digital camera with no particular post-processing) are indicated as Low Dynamic Range (LDR) images (Reinhard et al., 2010a). Their intrinsic dynamic limitation lead to unsatisfactory digital images when the real scene is intrinsically characterized by an high dynamic, such as for example when a camera frames an interior scene (with a very low light level), including a window showing a sunny daylight panorama (with a very high light level). In that case only one zone at a time can be properly imaged, while the other one lose its details (i.e. interior details hidden in the dark if exposure set on the exterior, or exterior details hidden in the white if exposure set on the interior).

\footnotetext{
${ }^{*}$ Corresponding Author
} 
For overcoming this problem High Dynamic Range (HDR) imaging has been developed starting from the late ' $90 \mathrm{~s}$. In this case multiple photographs with different exposure times are combined into a radiance map, which reflects the radiance in real-life scenes. The technique (Debevec and Malik, 1997), is based on multiple shots of a scene from the same position with exposure bracketing. If the images are taken at 8 bit per component (e.g. like when a jpeg output is chosen), 7 shots taken at 1 stop of difference (i.e. $-3,-2,-1,0,+1,+2,+3)$ are required for an optimal result (Barakat et al. 2008). From the integer RGB components of each image a floating point luminance can be estimated through different algorithms (Mann and Picard 1995, Debevec and Malik 1997, Robertson et al. 1999, De Neve 2009), generating luminance values ranging from $10^{-4}$ to $10^{8}$. However, current Digital Single Lens Reflex (DSLR) cameras allow to store images in raw mode, saving the whole 12 or 14 bits of information for each RGB channel that the camera is capable to acquire. In this case the 7 eight bit shots can be substituted by 3 "richer" shots spaced 2 stops apart $(-2,0,+2)$, producing nearly the same result (Barakat et al., 2008).

The opportunity to work with HDR images has never been systematically exploited in photogrammetry, and no imagebased modeling package is nowadays capable to process directly HDR radiance maps. However the process can be applied to 8 bit jpegs resulting from the tone mapping of floating points HDRs. Tone-mapping operators may compress the dynamic range of images by applying the same function to all pixels, or they may compress pixels based on their value and the values of a local neighborhood of pixels. As a practical result, details hidden in the brightest and darkest areas of each image due to clipping to the maximum $(255,255,255$, or white) and the minimum $(0,0,0$, or black) can be shown, allowing image matching to work properly also in areas otherwise not useful for generating geometrical data.

Although this principle seems rather evident, very few works have been published till now on the use of HDR in photogrammetry, with the exception of a validation in a laboratory experiment (Cai 2013) an application to roadway environments (Cai and $\mathrm{Li}$ 2014) and a general analysis for applications to strongly different illuminated Cultural Heritage structures (Ntregka et al, 2013).

What is instead usually applied in traditional photogrammetric applications for trying to enhance details in the darker and lighter areas of images is the Wallis filter. It is a digital processing function applied to the captured image that locally enhances the contrast levels, and is often applied in order to optimize images for subsequent image matching (Balstavistas, 1991). As the HDR processing, the Wallis filter works on the image luminance (not on colors) and its purpose might seem similar giving in general very good results with much less effort than HDR (Jazayeri and Fraser, 2008). However, it has to be noticed that the processing is applied to LDR images, and, if some details are hidden due to light intensity on the scene lower to the minimal recordable value or higher than saturation like with the very difficult materials that we are considering in this paper (e.g. an area of the luminance image containing an highlight, represented by a matrix of pixels all set to 255), no information can be re-extracted with any filtering. HDR processing overcomes this problem, operating somehow before this stage, saving the whole dynamic content of the image. In an ideal processing pipeline these two processes (HDR and Wallis filtering) could be even applied in a sequence, operating at different points of the whole process.

\section{PURPOSE OF THIS WORK}

Automated photogrammetry packages providing dense clouds of $3 \mathrm{D}$ points are everyday more diffused in both professional and research applications due to their capability of reducing the processing time, giving as results texturized $3 \mathrm{D}$ models with a relatively small effort, whose accuracy and precision level is satisfactory for many applications such as $3 \mathrm{D}$ imaging on Cultural Heritage (Fassi et al., 2013).

This category of programs are based on the approach known as structure from motion (SFM). The extraction and matching of features are usually carried out with the SIFT detector. Subsequently the external image parameters are solved by a bundle adjustment, providing both orientations and calibrations of the various images. Finally the imaged are matched generating a dense cloud of 3D colored points (GonzálezAguilera et al., 2012; Remondino et al., 2013). However not many settings are available for the operator since most of the process is automatic. It was decided to experiment the use of image pre-processing for gaining information in the brighter and darker areas of images devoted to image matching, both with optical means (i.e. the of polarizing filters) and digital means (i.e. HDR and tone mapping), with the purpose of facilitating the automatic orientation process and the following image matching, testing the results with real Cultural Heritage objects typically difficult to be captured with automatic photogrammetry procedures due to their shiny behavior.

The automated photogrammetry package used in this study was AGISOFT Photoscan and the point clouds generated by this software were compared numerically among each other to see the difference in terms of number of matched points with and without the analyzed pre-processing, for the same image orientation. For such comparison purpose it was decided not to complete an entire 3D acquisition, but just orient and match a minimal number of images for comparing the effects of the different pre-processing analyzed. This minimal number has been arbitrarily set to three.

\subsection{Test objects}

The six objects chosen for this test are deliberately difficult to be captured being made of materials typically unsuitable for automatic photogrammetry such as are metal and ceramic. In detail we used: i) a Roman decorated silver plate called "patera". It has a diameter of $17 \mathrm{~cm}$ and seemed to be a perfect object for this test due to its shiny surface in addition to its decoration with a lot of little details in relief; ii) a Mediaeval metal plate decorating a shield ("umbo"). The umbo chosen for the test (Fig. 3b) is made of metal with bronze plates decorating the flat part all around the objects and the tip of it.; iii) a silver box shaped as an apple, with the body close to a perfectly reflective surface with the exclusion of a few oxidation, and a more opaque leaf in the upper its side; iv) two dark and shiny Greek vases; iv) an Etruscan jug ("olpe"). Those object belongs to the Archaeological Museum of Milan (MAM) and to a private collection. The choice of such specific objects was determined by their dark and/or reflective surfaces generating highlights and dark areas.

The intention was to assess the above mentioned optical and digital image pre-processing for checking their effects on the creation of dense 3D point clouds through SFM/Image Matching seen as a whole "black box" process, as basically is for those operators working in the field of Cultural Heritage and using commercial automatic software packages. 


\subsection{Survey plan}

The survey for acquiring the images for photogrammetry was organized as in Fig. 1: the test objects were placed on a table with a monochromatic background. Three different camera positions (left, centre, right) were chosen, from which sets of three raw images have have been acquired. The first set without the filter and the second one with it, mounted on the lens and properly rotated for minimizing the highlights on the object surface.

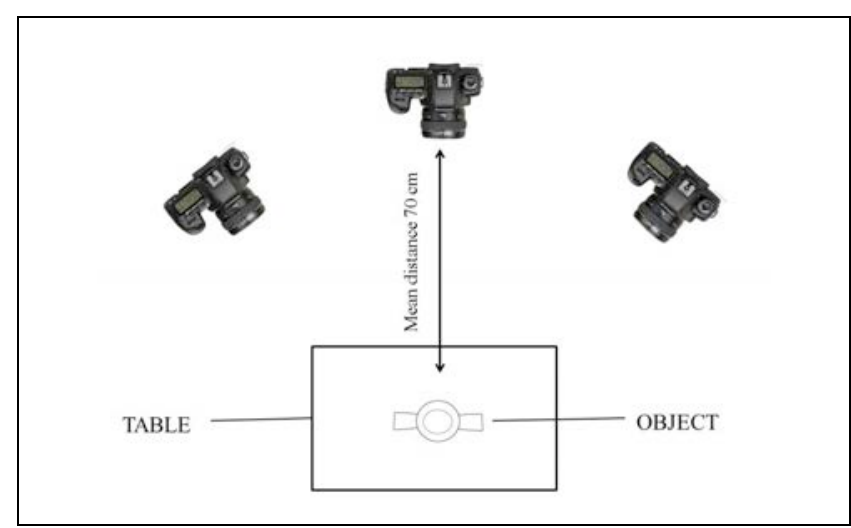

Figure 1. Set arrangement for the photogrammetric survey.

In front of the objects two coded targets at a known distance were placed to scale the point clouds after the processing. The set was placed on the footpath right outside the Museum in order to use the natural light. The shot were done during a cloudy day to avoid more reflections due to the sunlight. In this way, it was possible to use the same image for the processing with and without the filter, also with HDR images, so that the condition of light didn't change and the images were similar for each position. The camera used was a full frame CMOS sensor Canon 5D Mark II of 21 megapixel coupled with a $50 \mathrm{~mm}$ lens, placed on a tripod. The parameters of the shots were: ISO 200, the aperture on $f / 32$ to have the maximum depth of field, manual focusing and the exposure controlled so that with the filter mounted on, the difference was always of 1.5 stop in respect to the images without the filter. The mean distance between the camera and the objects was around $70 \mathrm{~cm}$. The dimensions of the images were $5616 \times 3744$.

\section{ACQUISITION AND PROCESSING}

As mentioned above the camera positioning was the same for each object. Two set of three images were shot from each position, the first without the and the second with the polarizing filter mounted on the lens. All images were acquired in raw mode in order to maintain all the 14 bit of information per RGB component. The three 14 bit LDR images were merged afterwards in the lab through a commercial HDR package (Photoshop by Adobe software, function "merge to HDR Pro") in a single 32 bit HDR image. Each HDR was then tone mapped to an 8 bit image. This step is potentially critical since several mapping approach have been developed such as "Local Adaptation", "Equalize Histogram", "Exposure and Gamma" and "Highlight Compression" (Venkata Lakshmi, 2012). We chose "Equalize Histogram" being the process that allows to locally enhance the image contrast. In addition the other possible mappings have several possible input parameters that would have complicated the results comparison.

The Ground Sampling Distance (GSD) was kept below $0.11 \mathrm{~mm}$. Specifically the different objects represented in fig. 3 where captured at an distance/GSD reported in the following table. Being object not flat these figures represent just average values.

\begin{tabular}{|l|c|c|}
\cline { 2 - 3 } \multicolumn{1}{c|}{} & \multicolumn{1}{c|}{$\begin{array}{c}\text { Shooting } \\
\text { distance }(\mathrm{cm})\end{array}$} & $\begin{array}{c}\text { Average } \\
\text { GSD }(\mu \mathrm{m})\end{array}$ \\
\hline Patera (fig 3a) & 80 & 102 \\
\hline Umbo (Fig 3b) & 51 & 65 \\
\hline Apple (Fig. 3c) & 58 & 74 \\
\hline Greek vase (Fig. 3d) & 69 & 88 \\
\hline Etruscan Olpe (Fig. 3e) & 74 & 95 \\
\hline Greek vase (Fig. 3f) & 66 & 85 \\
\hline
\end{tabular}

Table 1. Shooting distances and average GSDs.

For the data processing Agisoft Photoscan was used because of its massive and successful usage for 3D modeling in the Cultural Heritage field. This software at the first stage detects points in the source photos in a similar way of the well-known SIFT approach and generates a descriptor for each point based on its local neighborhood. These descriptors are used later to detect correspondences across the photos. For solving camera intrinsic and extrinsic orientation parameters, the software uses a greedy algorithm to find approximate camera locations and refines them later using a bundle-adjustment algorithm.
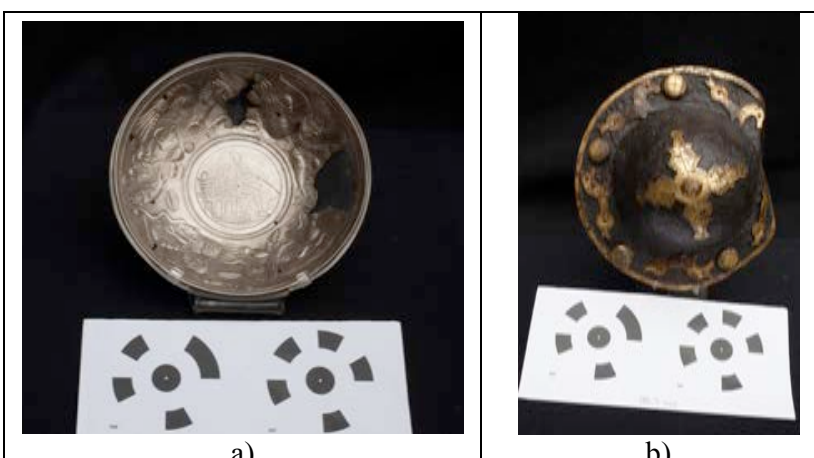

a)
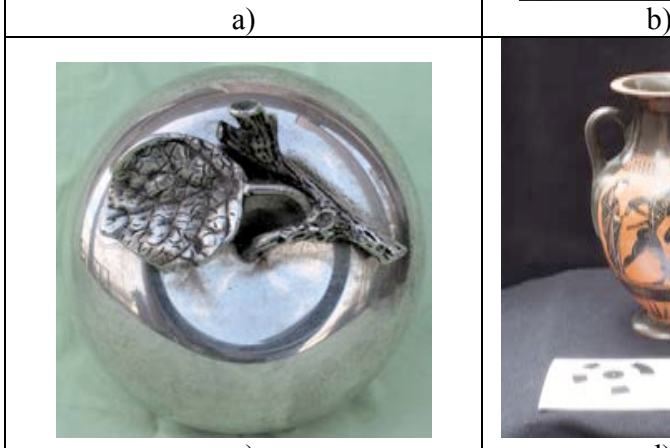

c)

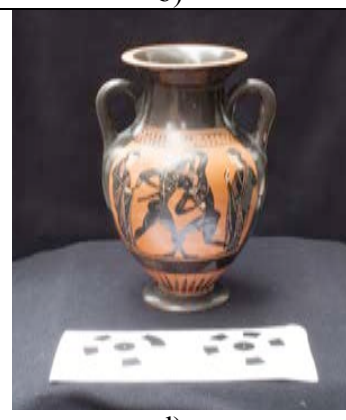

d)

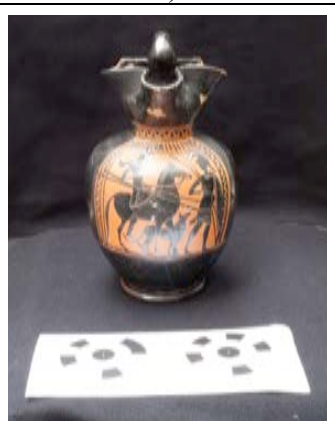

e)

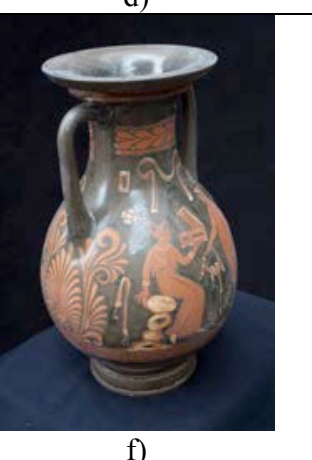

Figure 3. Metallic and ceramic test objects used in the experiments: a) Patera del Pescatore (metal); b) Lombard Umbo (metal); c) Apple box (metal), d) Greek vase (ceramic); e) Etruscan olpe (ceramic); c) dark Greek vase (ceramic). 
Three vases were chosen for their intrinsic characteristics, as the presence of large parts of black painting that produced textureless and shiny surfaces. It was decided to use all vases, even if the structures are quite the same, because of their colors and geometry. The first Greek vase (Fig. 4a) is a black on red coloured amphora dating back to the IV century BC while the Etruscan vase (Fig. 4b) is a black on red colored olpe (wine jug) dating back to the V/IV century BC. As shown in figure the Etruscan vase has a very small radius of curvature between the body and the collar, meaning that in that particular part of the vase the reflections were augmented because of the shape.

An additional Greek pieces, a very dark vase (Fig. 4c) was used in a successive test for comparing the effects of HDR generated in simplified way from a single 14 bit raw image (used for the experiment on the first two vases), against a "true" HDR originated from a triplet of raw images with exposures spaced 2 stops apart $(-2,0,+2)$.

\section{RESULTS AND DISCUSSION}

Each triplet of images was easily masked using the dark background as selection level. The masks were then exported in order to use them also for all the images acquired from a certain position with the different levels of image preprocessing (no filter; filter; no HDR; HDR). Both orientation and image matching were then launched on masked images. The resulting indexes such as the number of tie points automatically evaluated during orientation, and the number of points actually matched for generating the dense 3D cloud were saved and compared each other.

The targets shown in both figures 3 and 4 were used to metrically resize the model, but they resulted also helpful during the alignment phase. However they have been removed from the process while producing the dense point cloud using the "aggressive" depth filtering. The Patera, the Umbo and the first two vases were used for analyzing the effect of polarizing filters, and the image matching results are reported in tables 2 to 5.

\begin{tabular}{|l|c|c|}
\cline { 2 - 3 } \multicolumn{1}{c|}{} & Tie points & Matched points \\
\hline No filter & 3795 & 920306 \\
\hline Polarizer & 3959 & 913688 \\
\hline
\end{tabular}

Table 2. Metallic object \#1 (Patera). Orientation and matching results with and without polarizing filter.

\begin{tabular}{|l|c|c|}
\cline { 2 - 3 } \multicolumn{1}{c|}{} & Tie points & Matched points \\
\hline No filter & 4899 & 1470523 \\
\hline Polarizer & 4824 & 1416145 \\
\hline
\end{tabular}

Table 3. Metallic object \#2 (Umbo). Matching results with and without polarizing filter.

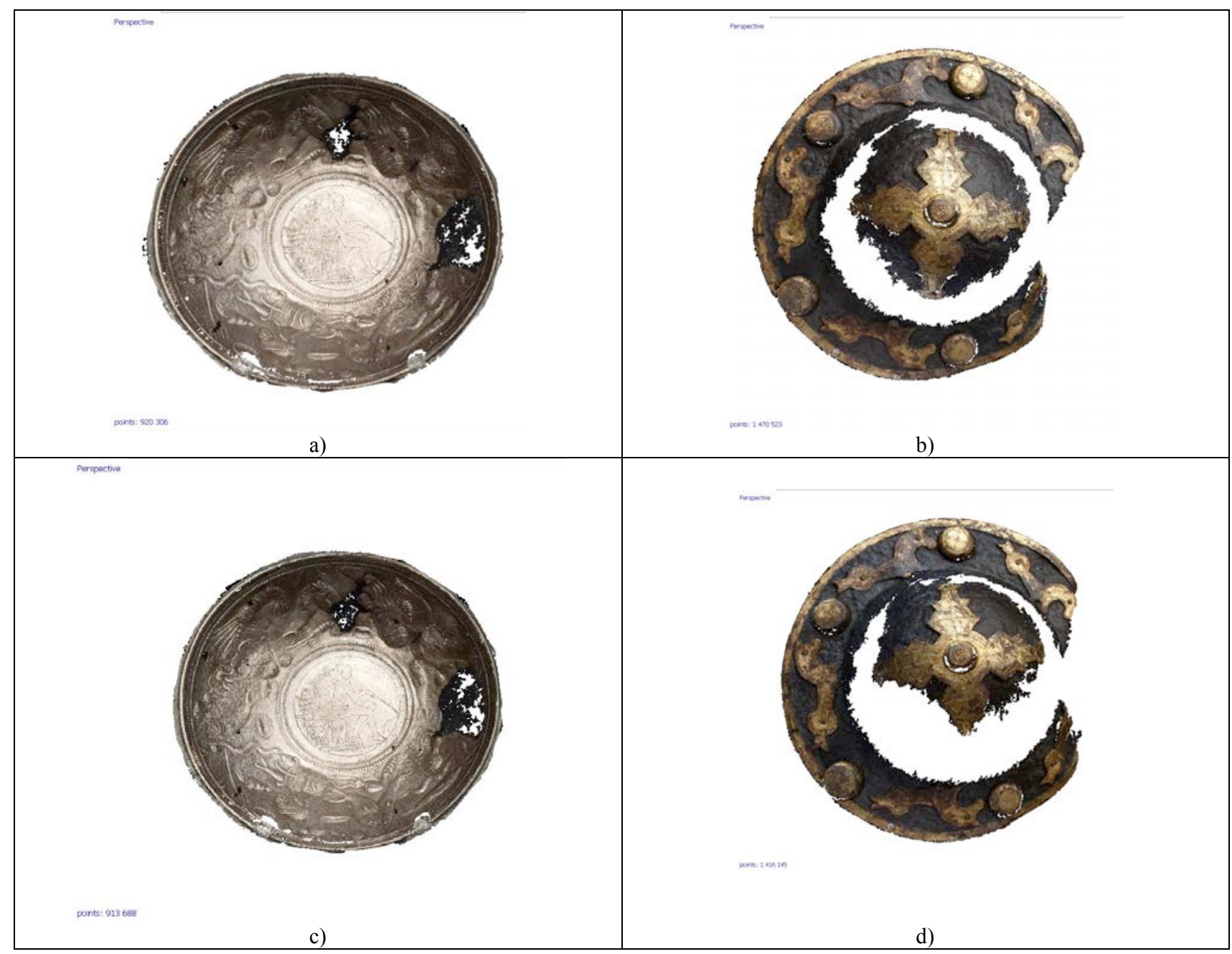

Figure 5. Image matching results on the metallic test objects with and without polarizing filter: a, c) Patera; b, d) Umbo. The first row $(a, b)$ acquired without any polarizing filter while the second $(c, d)$ with polarizing filter. The two results show no significant difference. 


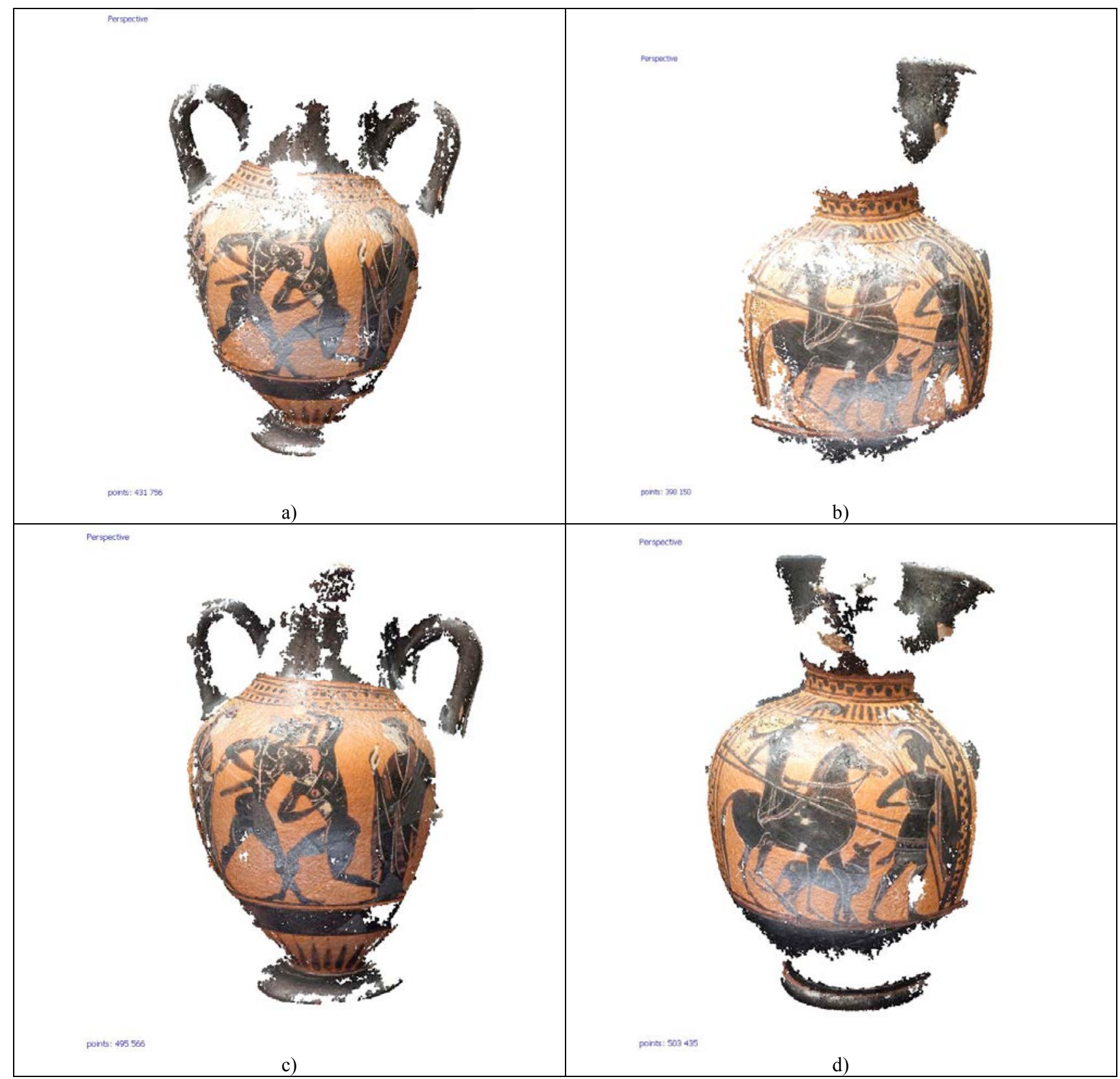

Figure 6. Image matching results on the ceramic test objects with and without polarizing filter: a, c) Greek vase; b, d) Etruscan vase. The first row $(a, b)$ acquired without any polarizing filter while the second $(c, d)$ with polarizing filter.

\begin{tabular}{|l|c|c|}
\cline { 2 - 3 } \multicolumn{1}{c|}{} & Tie points & Matched points \\
\hline No filter & 1424 & 431756 \\
\hline Polarizer & 1389 & 495566 \\
\hline
\end{tabular}

Table 4. Ceramic object \#1 (Greek vase). Matching results for different image pre-processing

\begin{tabular}{|l|c|c|}
\cline { 2 - 3 } \multicolumn{1}{c|}{} & Tie points & Matched points \\
\hline No filter & 1108 & 399387 \\
\hline Polarizer & 1169 & 503435 \\
\hline
\end{tabular}

Table 5. Ceramic object \#2 (Etruscan olpe). Matching results for different image pre-processing
From figure 5 it possible to see qualitatively that polarizers do not affect image matching results. By analysing the two tables 2 and 3 we can see, as theoretically expected, that for both metallic objects the polarizing filters do not give improvements for the physical behaviour of electromagnetic waves on metallic surfaces. This involves that light reflections from metallic surfaces are not polarized and the related highlights can't be attenuated with any possible rotation of the filter. As a result both table 2 and table 3 show a number of tie points and matched points, lower when using the filter than without filter. Differently for the shiny patera, the umbo is darker but also more opaque. Hence, image matching works generally better than in the previous case. In particular, due to the lower dynamic range between highlights and dark parts, in this case the matching of images gives better results with HDR processing rather than without any processing, with an increase of matched points from 1470523 to 1572164 (+7\%) without 
polarizing filter, and from 1416145 to $1487364(+5 \%)$ with the polarizing filter on.

With the ceramic objects the polarizing filters becomes much more important, since the physical behaviour of ceramic materials allows electromagnetic waves to keep their "natural" polarization. Reflections can be therefore strongly reduced thanks to filtering, and the increased number of matchable details allows to rise the number of matched points. This behaviour is shown in figure 6 , where the second row of images qualitatively shows a number of matched points larger that the images of the first row, taken without polarizer. This qualitative impression is confirmed by numbers in tables 4 and 5 . Tables 4 shows an increase on matched points from 431756 to 495566 $(+15 \%)$ for the Greek vase whose images have been taken respectively without and with polarizing filter and no HDR. Also Table 5 shows and increase of matched points from 399387 to 503435 for the Etruscan olpe, corresponding to a considerable $+26 \%$.

However no influence seems to have HDR, at list using the single shot tone mapping used for all items considered till now, that in some cases seems, as witnessed by the numbers in tables $2-5$, to give results worst than those generated without HDR processing. In order to check if such absence of increase in SFM performances is due to the way HDR have been implemented, a new set of images have been done for the Greek vase of figure 4c, whose HDR radiance maps have been calculated starting from exposure bracketing, as shown in figure 7 .

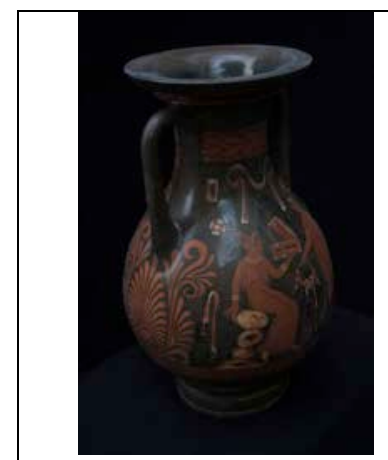

a)

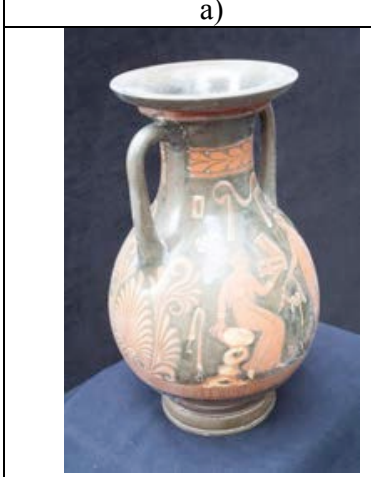

c)

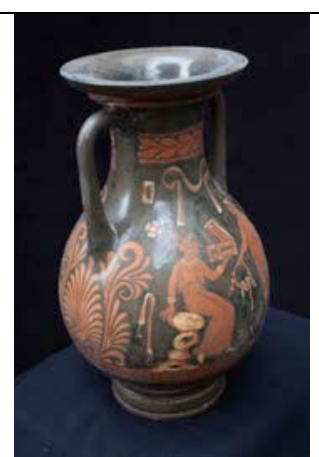

$\mathrm{b}($

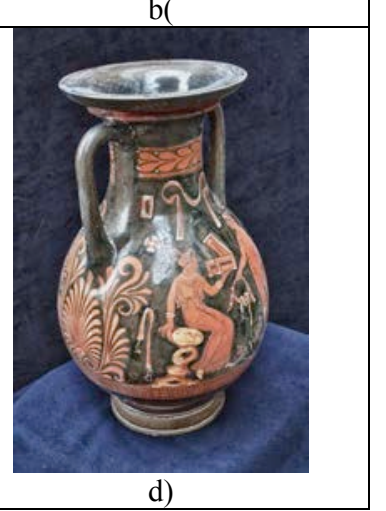

Figure 7. Canonical HDR processing with 3 raw shots at different exposures: a) -2 stops; b) right exposure; c) +2 stops; d) tone mapping of the resulting HDR image.

Once the images properly exposed (like that in Fig. 7b), and the images resulting from the tone mapping of a "true" HDR image (like that in Fig. 7d) are matched, the result is the one described in figure 8 and table 5 .

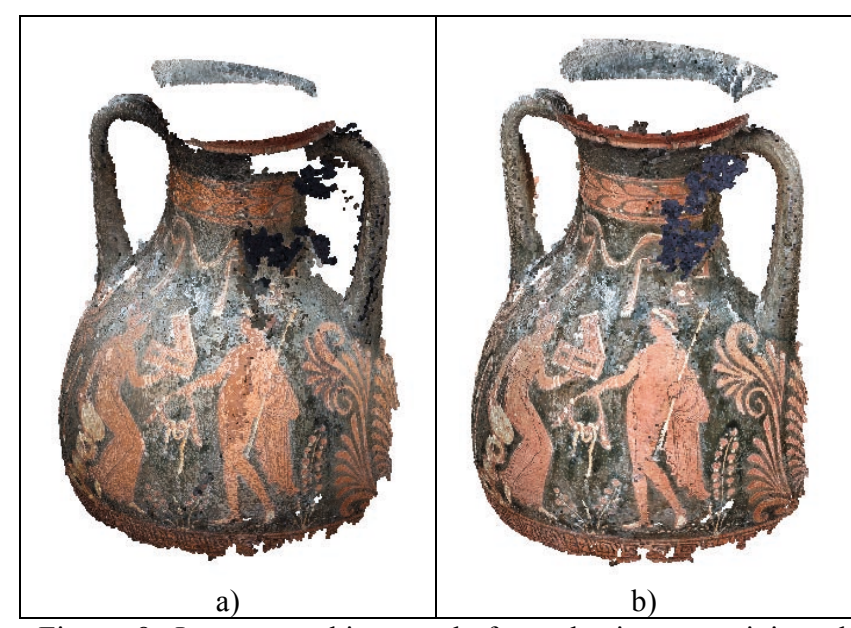

Figure 8. Image matching result from the images originated with different HDR processing: a) No HDR; b) tone mapped images from HDR processing.

From Fig. 8 it is possible to see qualitatively a small increase in the number of matched points, specially in the most "difficult" areas, like the dark internal area of the vase neck, and a few points in the exterior part of it, evident in the upper right sector of Fig 8a, that in Fig 8b are properly covered. In quantitative terms table 6 shows an increase from 5637561 to 5858700 matched points, corresponding to $4 \%$. Such value is not so large but we have to consider that such small amount of additional points is formed by all those "difficult" points that would have never been captured with standard imaging due to their darkness with respect to the environment.

\begin{tabular}{|l|c|c|}
\cline { 2 - 3 } \multicolumn{1}{c|}{} & Tie points & Matched points \\
\hline LDR & 5467 & 5637561 \\
\hline Tone mapped HDR & 5646 & 5858700 \\
\hline
\end{tabular}

Table 6. Ceramic object \#3 (dark Greek vase). Matching results for different image pre-processing and no polarizer filtering.

A similar comparison has been done with the silver apple shown in Fig. 3c, providing a far better result, shown qualitatively in the following image.

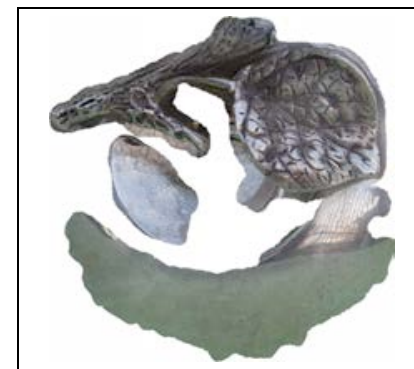

a)

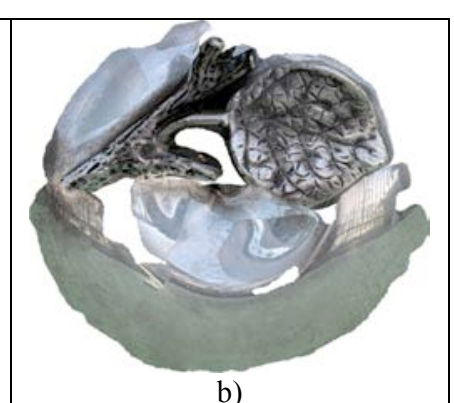

b)
Figure 8. Image matching result from the silver apple images originated with different HDR processing: a) No HDR; b) tone mapped images from HDR processing.

In this latter case both the SFM orientation and the image matching phase are rather difficult due to the high specularity of the imaged object that generated strong highlights saturating portions of the images. This is confirmed by the small numbers of tie points detected on LDR images (table 7). Once the HDR processing is applied the details contained in such image areas are recovered, allowing both SFM orientation and image matching to work better, producing as a final result a 
significative increase in the matched points from 627,256 to $1,024,126(+63 \%)$.

\begin{tabular}{|l|c|c|}
\cline { 2 - 3 } \multicolumn{1}{c|}{} & Tie points & Matched points \\
\hline LDR & 898 & 627256 \\
\hline Tone mapped HDR & 1030 & 1024126 \\
\hline
\end{tabular}

Table 7. Third metallic object (silver apple) matching results for different image pre-processing.

\section{CONCLUSION}

The use of processing for compressing the dynamic range of high-contrast images originated by object with dark or highly reflective materials, has been shown. Such compression aims at improving the matching of images in the framework of the SFM/image matching 3D modeling pipeline.

The analyzed tools are: i) at optical level the strong attenuation (or the complete cancellation, for proper angles) of polarized light components influencing negatively Image Matching algorithms with fake matchings, originating wrong or imprecise clouds of 3D points; ii) at digital level the use of HDR/tone mapping processing, for obtaining details in those areas of the images, generally saturated by over-exposition or underexpositions, whose matching is intrinsically erroneous due to the absence of information to be matched.

Such tools have been discussed and applied to specific and critical Cultural Heritage applications, referred to the 3D acquisition and modeling of some dark and shiny archaeological remains made of different materials.

The main results are that polarizing filters may greatly help in improving image quality automatic photogrammetry with all those material such as ceramic, that is capable to maintain the natural polarization of light. The experimental result make evident an improvement from $5 \%$ to $25 \%$ of the matched points after filtering. However metallic object do not conserve light polarizations and are consequently not affected by such enhancement.

HDR has also been tested revealing a moderate enhancement on the tested ceramic object, in the order of $5 \%$, with respect of standard images, but a definitely much better result on a metallic object $(+63 \%)$.

\section{REFERENCES}

\section{References from Journals:}

Barakat N., Hone A.N., Darcie T.E., 2008. Minimal-Bracketing Sets for High-Dynamic-Range Image Capture, IEEE Transactions on Image Processing, vol. 17, no. 10, pp. 18641875.

Cai, H., 2013. High Dynamic Range Photogrammetry for Light and Geometry Measurement. AEI, pp. 544-553.

Cai, H., Li, L., 2014. Measuring Light and Geometry Data of Roadway Environments with a Camera, Journal of Transportation Technologies, 4, pp. 44-62.

Patrick Ledda, Alan Chalmers, Tom Troscianko, Helge Seetzen, 2005. Evaluation of tone mapping operators using a high dynamic range display, ACM Transactions on Graphics 24 (3), pp. 640-648.

Robertson, M.A., Borman, S., Stevenson, R. L., Estimationtheoretic approach to dynamic range enhancement using multiple exposures, Journal of Electronic Imaging, 12(2), 2013, pp. 219-228.

Yoshida, A., Mantiuk, R., Myszkowski, K, Seidel, H-P, 2006. Analysis of reproducing real-world appearance on displays of varying dynamic range, Computer Graphics Forum 25 (3) pp. $415-426$.

\section{References from Books:}

Feininger, A., 1954. Successful Photography, Prentice Hall, Englewood Cliffs, NJ, USA, pp. 70-72.

Goldberg, N., 1992. Camera Technology: The Dark Side of the Lens. Academic Press. pp. 141-147. ISBN 978-0-12-287570-0.

Holst, G.C., CCD Arrays, Cameras, and Displays, JCD Publishing and SPIE Optical Engineering Press, Bellingham, WA (1996), pag. 113.

Paine, D.P., Kise, J.D., 2012. Aerial Photography and Image Interpretation, John Wiley \& Sons, Hoboken NJ, USA.

Reinhard, E., Ward, G., Pattanaik, S., Debevec, P., 2006. High Dynamic Range Imaging, Morgan Kaufmann, Burlington, MA, USA, Chapter 03 - High Dynamic Range Image Encodings, pp. 91-93.

Reinhard, E., Ward, G., Pattanaik, S., Debevec, P., 2006. High Dynamic Range Imaging, Morgan Kaufmann, Burlington, MA, USA, Chapter 07 - Spatial Tone Reproduction, pp. 223-323.

\section{References from Other Literature:}

De Neve, S., Goossens, B., Luong, H., Philips, W., 2009. An improved HDR image synthesis algorithm, 16th IEEE International Conference on Image Processing (ICIP), pp. 1545,1548 .

Debevec P. E., Malik J., 1997. Recovering High Dynamic Range Radiance Maps from Photographs, SIGGRAPH '97, Proceedings of the 24th annual conference on Computer graphics and interactive techniques, pp. 369-378.

Fassi, F., Fregonese, L., Ackermann, S., De Troia, V., Comparison between laser scanning and automated 3D modelling techniques to reconstruct complex and extensive cultural heritage areas, ISPRS Archives, Vol. XL-5/W1, 2013, pp. $73-80$.

González-Aguilera, D., Fernández-Hernández, J., ManceraTaboada, J., Rodríguez-Gonzálvez, P., Hernández- López, D., Felipe-García, B., Gozalo-Sanz, I., Arias-Perez, B., 3D modelling and accuracy assessment of granite quarry using unmmanned aerial vehicle, ISPRS Annals of the Photogrammetry, Remote Sensing and Spatial Information Sciences 07/2012; I(3):37-42.

Jazayeri, I., Fraser, C.S., Interest Operators in Close-Range Object Reconstruction, ISPRS Archives, Vol. XXXVII Part B5, 2008, pp. 69-74 
Mann, S. , 1993. Compositing multiple pictures of the same scene, Proc. 46th Annual IS\&T Conf., Boston, MA, May 9-14, Vol. 2, pp. 50-52.

Mann, S. and Picard, R.W., 1995. On being 'undigital' with digital cameras: Extending dynamic range by combining differently exposed pictures, IS\&T's 48th Annual Conf., Washington, D.C., pp. 422-428, May 7-11.

Medina, Rachel A., Scappaticci, Nicholas I., and Bodenbender, Brian E., 2011. High Dynamic Range Images in Sand Dune Photogrammetry. Geological Society of America, Abstracts with Programs, Volume 43, Issue 5, January 1, p.433

Ntregka, A., Georgopoulos, A., Santana Quintero, M., 2013. Photogrammetric Exploitation of HDR Images for Cultural Heritage Documentation, ISPRS Annals of the Photogrammetry, Remote Sensing and Spatial Information Sciences, Volume II5/W1, XXIV International CIPA Symposium, 2 - 6 September 2013, Strasbourg, France, pp. 209-214.

Remondino, F., Spera, M.G., Nocerino, E., Menna, F., Nex, F., Gonizzi-Barsanti, S., Dense image matching: Comparisons and analyses, Digital Heritage International Congress (DigitalHeritage), 2013, vol. 1, pp.47-54, doi: 10.1109/DigitalHeritage.2013.6743712

Robertson M. A., Borman S., Stevenson R. L., 1999. Dynamic range improvement through multiple exposures, Int. Conf. Image Process., Vol. 3, pp. 159-163.

Venkata Lakshmi, S.; Sujatha, S.; Janet, J.; Bellarmine, T., Analysis of tone mapping operators on high dynamic range images, Proc. of IEEE Southeastcon, 2012, pp.1-6. doi: 10.1109/SECon.2012.6196908

Wells, J. M., Jones, T. W., Danehy, P. M., 2005. Polarization and color filtering applied to enhance photogrammetric measurements of reflective surfaces, $46^{\text {th }}$ AIAA/ASME/ASCE/ASC Structures, Structural Dynamics \&Materials Conference, 18-21 April 2005, Austin, Texas, pp. 1887-1896. 